\title{
Disrupting Experimental Strategies for Inhalation Toxicology: The Emergence of Microengineered Breathing-Smoking Human Lung-on-a-Chip
}

\author{
Kambez H. Benam ${ }^{1,2}$
}

\begin{abstract}
Cigarette smoking is major public health problem and a substantial contributor to the pathogenesis of respiratory disorders such as chronic obstructive pulmonary disease and lung cancer. In addition, our society faces the rapidly growing use of tobacco-related products such as electronic cigarettes. Thus, we need reliable model systems that can accurately predict the biological impact of exposure to inhaled smoke and vape and allow recreation of clinically relevant organ-level responses. In this study, we describe the emergence of "Breathing-Smoking Human Lung-on-a-Chip" microdevice at the interface of tissue microengineering, pulmonary biology, toxicopathology, and systems biology to enable study of smoke-induced lung injuries with high fidelity and precision in vitro. We also address the ability of this novel microfluidic system to overcome several major limitations of widely used animal models and static in vitro cultures of smoke exposure. While at its infancy, this technological advancement offers significant potential on how experimental inhalation toxicity studies can be performed.
\end{abstract}

Keywords: breathing-smoking human lung-on-a-chip, electronic cigarettes, inhalation toxicology, predictive modeling, small airway-on-a-chip, tobacco cigarettes

$\mathbf{O}$ VER THE PAST FEW YEARS, the interplay of costs and ethics and, importantly, the need for enhanced pathophysiological relevance have drastically paved the path for development of reliable model systems that more accurately mimic biological responses in humans. Currently, many toxicology studies cannot be performed directly on human subjects. Therefore, animal testing and static in vitro culture systems have become routine predictive models in many laboratory settings. However, key anatomical and physiological differences between humans and animals and inability of 2D cell cultures to reproduce complex organ-level functionalities and recreate dynamically interacting multicellular structures make it very difficult to extrapolate the findings to humans. Furthermore, there is a growing ethical concern to reduce the use of animals in preclinical studies.

In this study, we discuss how recent advances in tissue microengineering have enabled development of microfluidic Lung-on-a-Chip devices that simulate human lung pathophysiology and allow study of inhalation toxicity, with a particular focus on cigarette smoke exposure.

\section{Inhalation Toxicology and Cigarette Smoke Exposure}

Breathing generates the greatest total exposure of humans to environmental contaminants. Human adults on average in- hale $10,000-20,000 \mathrm{~L}$ of air within 24 hours, as opposed to $\sim 1.5$ and $0.6 \mathrm{~L}$ of exposure to liquid/water and food intake via digestive system, respectively. ${ }^{1}$ Given the large daily intake and almost complete absence of pollutant-free air in our environment, it is critical that we are able to characterize and predict biological impact of exposure to gases, vapors, and aerosols in the inhaled air. Moreover, it is imperative to take into account appropriate considerations when modeling or evaluating exposure to airborne contaminants in active versus passive inhalation and clinical translation of the findings.

The inhalation toxicity of cigarette smoke is a major public health problem and substantially contributes to the pathogenesis of respiratory disorders in particular chronic obstructive pulmonary disease (COPD) - currently the third leading cause of death in the United States with rising incidence in morbidity and hospitalization ${ }^{2,3}$ and lung cancerthe primary cause of cancer-related mortalities worldwide. ${ }^{4}$ Thus, we need reliable and predictive model systems that can enable accurate and human-relevant analysis of cigarette smoke-induced toxicity and lung injury. In addition, the rapidly growing popularity of tobacco-related products such as electronic cigarettes (ECs) further necessitates development and application of such models to better characterize

\footnotetext{
${ }^{1}$ Division of Pulmonary Sciences and Critical Care Medicine, Department of Medicine, University of Colorado Denver, Aurora, Colorado.

${ }^{2}$ Department of Bioengineering, University of Colorado Denver, Aurora, Colorado.
} 
biological impact of EC emissions on the lung, which is a high-priority health concern for regulatory agencies. ${ }^{5,6}$

\section{Commonly used model systems and their challenges}

Laboratory animal models, especially rodents, have been frequently used for inhalation exposure to aerosols, gases, and infective agents. But, considerable interspecies differences with humans in histology, anatomy, and organ architecture of the lungs as well as cellular distribution in the immune compartment and the route of exposure to airborne stimuli challenge their suitability to faithfully mimic human response regardless of the type of airborne material. Rats and mice, for example, have less extensive airway branching and $\sim 10$-fold greater number of nonciliated club-like cells and less mucin-producing cells in their small airways, which is a major site of damage and inflammation in the respiratory tree, compared with humans. ${ }^{7}$

In addition, cellular distribution of immune compartment in the peripheral blood varies significantly between humans and rodents. For instance, neutrophils that rapidly mobilize out of bone marrow into bloodstream to localize to the lungs during acute infection, inflammation, and injury comprise only $10 \%-25 \%$ of circulating white blood cells in mice, whereas they represent $50 \%-70 \%$ in humans. ${ }^{8,9}$ Importantly, rodents are obligate nose breathers with intricate and highly developed nasal turbinates that lead to different particulate deposition patterns than humans. ${ }^{10}$ Therefore, in the context of cigarette smoke exposure, rodents at best are passive smokers and, unlike humans, are unable to actively inhale smoke and control smoking topography and breathing behavior. As such, they are often continually exposed to high doses of cig- arette smoke and require extensive handling and restraint, which do not recapitulate clinically relevant behavior of human smokers and is ethically a major concern.

Several in vitro models of lung epithelial cells exposure to cigarette smoke have been developed. ${ }^{11-19}$ However, a major limitation of these culture systems is that they are unable to reproduce exposure of well-differentiated mucociliated airway epithelium to aerosolized smoke under physiologically relevant inhalation-exhalation airflow conditions, which are responsible for delivering smoke to the lung mucosa.

The majority of the models expose the epithelial cells to cigarette smoke extract (CSE), which contains only the hydrophilic constituents of the cigarette smoke, ${ }^{11-13}$ or to cigarette smoke condensate (CSC), which simply is composed of the smoke hydrophobic particulate matter. ${ }^{14}$ In these circumstances, even if primary human mucociliary lung epithelia (as opposed to undifferentiated immortalized or cancerous cell lines) are used, the physiologically critical air-liquid interface (ALI) is lost due to temporary submersion of the cultures with CSE/CSC-containing media, and importantly a considerable proportion of the whole cigarette smoke (WCS) is absent.

WCS contains both hydrophobic and hydrophilic constituents in particulate and gaseous compartments. ${ }^{15-17}$ To induce the full complement of toxicities and pathologies associated with smoke-induced airway injury, as observed in human smokers, it is crucial to recreate exposure to the WCS in vitro. ${ }^{16,17}$ More recently, several studies have reported a method for generating, diluting, and delivering WCS to the lung epithelium cultured at ALI ${ }^{16-19}$; but, this approach only permits exposure of the epithelial cells to "vertically" delivered puffs of smoke under static conditions.

FIG. 1. Human Small Airway-on-a-Chip. (a, b) Schematic cartoon of the Small Airway Chip cross-section demonstrating airflow and microvascular channels as well as the porous membrane getting populated on opposite sides with primary human mucociliated pseudostratified bronchiolar epithelium and lung microvascular endothelium. (c) A 3D reconstruction of the epithelium (green)-endothelium (red) tissue-tissue interface that forms on-chip. (d) Tight junction formation in the well-differentiated human small airway epithelium in the chip (red: ZO1 immunostaining). (e) The endothelial monolayer formed on-chip is also continuously packed, as illustrated by PECAM-1 staining (green). (f) Pseudocolored scanning electron micrograph of cilia (magenta) and microvilli (cyan) on the apical surface of the differentiated bronchiolar cells formed onchip. (g) Modeling COPD exacerbation on-chip: graphs showing effects on production of the cytokines IL-8 and M-CSF by TLR stimulation with LPS (TLR-4 ligand; bacterial infection mimic) or poly I:C (TLR-3 ligand; viral infection mimic) in Small Airway Chips lined by bronchiolar epithelial cells obtained from healthy donors versus COPD patients. Note selective upregulation of cytokines in response to LPS versus poly I:C in healthy versus COPD. This enables validation of the model (e.g., in case of IL-8) and discovery of new biomarkers (e.g., virally [not bacterially]-induced M-CSF secretion from COPD [not healthy] airway epithelia). (h) Modeling asthma on-chip: graphic depictions of IL-13-induced (in healthy airway epithelia) increase in total culture area covered by goblet cells (Muc5AC positive immunostaining) and secretion of the cytokine G-CSF. (i) Sequential time-lapse microscopic views showing blood-extracted fresh neutrophils being recruited to the surface of living exacerbated endothelium, and rolling on the surface membrane, under physiological flow and shear stress of $1 \mathrm{dyne} / \mathrm{cm}^{2}$ ) in a chip when the epithelial cells had been stimulated with poly I:C. Yellow arrowhead shows a neutrophil that is being recruited from flow, directly adjacent to an already bound/recruited neutrophil. (j) Pharmacological modulation of COPD exacerbationassociated inflammation in the Small Airway-on-a-Chip: recruitment of neutrophils to the surface of endothelium in the Small Airway Chips containing a differentiated COPD epithelium with an underlying endothelium pretreated with corticosteroid Budesonide, lead preclinical therapeutic compound BRD-4 inhibitor, or DMSO (drug carrier; control) for 24 hours before poly I:C stimulation. The graph illustrates drug-mediated inhibition of neutrophil adhesion in exacerbated chips. Note the results compare the drug effect in Small Airway Chip system (black bars) against data obtained when similar studies were performed in static Transwell insert cultures (white bars). The microfluidic system was more relevant to capture the enhanced inhibitory effect of the BRD-4 inhibitor as this compound preferentially blocks early adhesion and rolling responses mediated by E-selectin and VCAM-1 that are required for initiation of the neutrophil recruitment cascade. ${ }^{9}$ Reproduced with modifications from Benam et al. ${ }^{9,24}$ with permission from Elsevier, Inc. (Cell Press) and Macmillan Publishers Ltd. (Springer Nature). BRD-4, bromodomain containing 4; COPD, chronic obstructive pulmonary disease; G-CSF, granulocyte colony-stimulating factor; IL-8, interleukin 8; LPS, lipopolysaccharide; M-CSF, macrophage colony-stimulating factor; PECAM-1, platelet and endothelial cell adhesion molecule 1; TLR, Toll-like receptor; VCAM-1, vascular cell adhesion molecule 1. 
Specifically, these systems fail to effectively reproduce exposure to the whole smoke under breathing airflow conditions that apply "horizontal" shear forces across the surface of the airway epithelium in vivo. ${ }^{20,21}$

\section{Organs-on-Chips}

Advances in tissue microengineering have made it possible to create biomimetic microfluidic cell culture devices, known as "organs-on-chips," that are the size of a flash memory drive and contain micrometer-sized hollow channels lined by living cells. These platforms are highly customizable and enable researchers to reproduce multicellular architecture, physiochemical microenvironment, tissue-tissue interface, and intercellular crosstalk of the human organs with an unprecedented fidelity and precision in vitro. Moreover, they allow recreation of critical dynamic processes that are observed in vivo such as vascular perfusion and leukocyte circulation. ${ }^{9,22-25}$ Thus, they offer new opportunities as advanced preclinical models for disease modeling, toxicopathological analysis, therapeutic target identification and validation, and biomarker discovery.

\section{Reverse Engineering Inhalation Pathophysiology Using a Microfluidic Breathing Human Lung Chip}

Small airways, also known as bronchioles, are noncartilaginous conducting airways with an internal diameter of $2 \mathrm{~mm}$ a

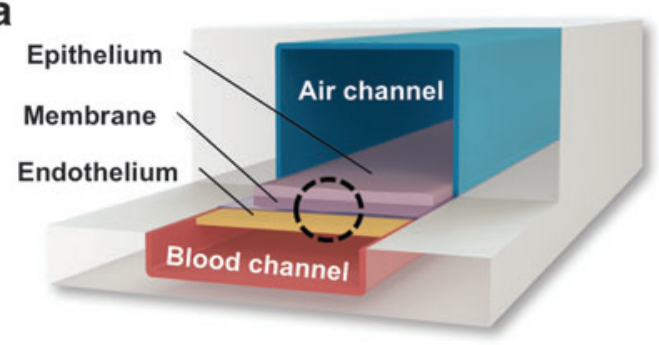

b

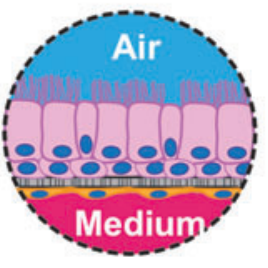

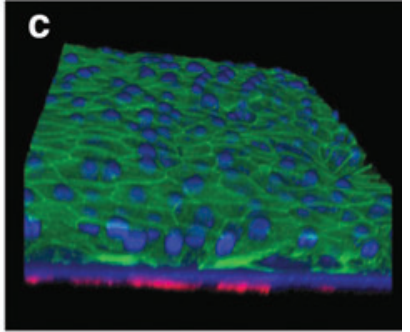
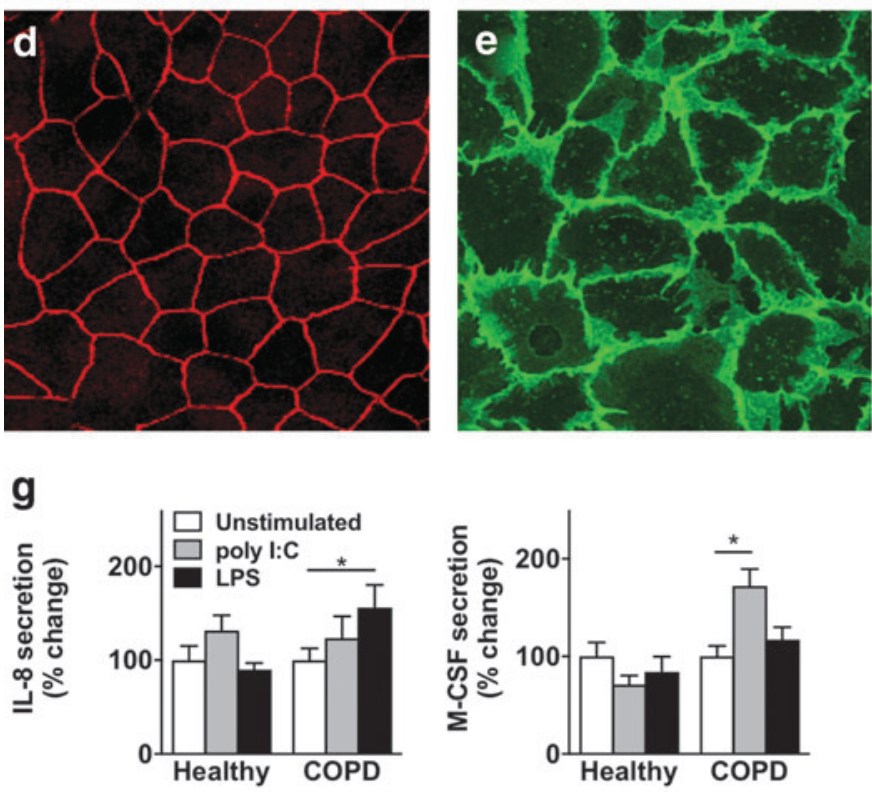

i

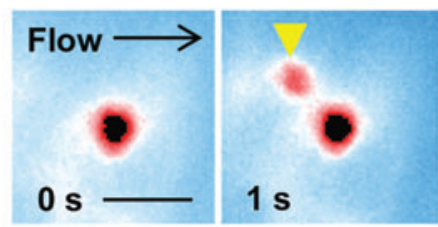

$2 \mathrm{~s}$

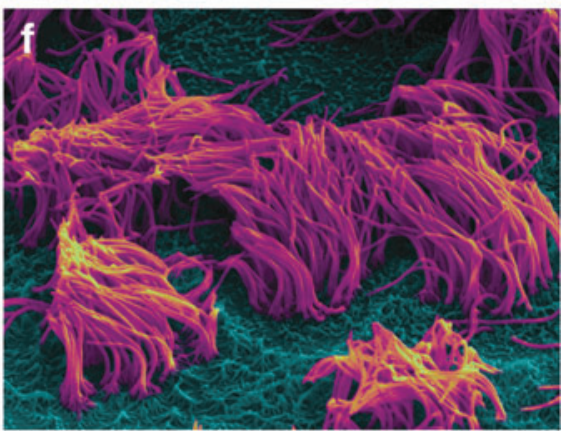

h
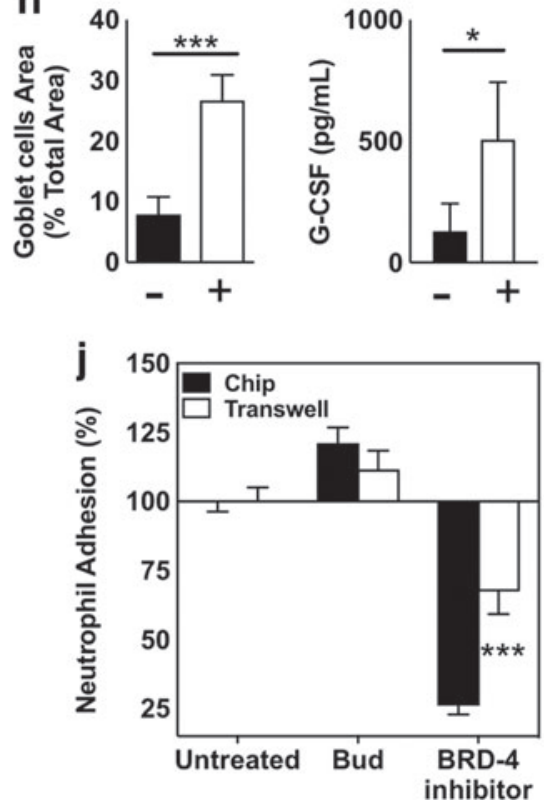
or less, which have been reported as key sites of pathology and airflow obstruction in a range of lung disorders, including COPD, asthma, idiopathic pulmonary fibrosis, and cystic fibrosis. ${ }^{26-29}$ Benam et al., recently developed a living microengineered mimetic of human bronchiole, which was made of a biocompatible and transparent polymer, called poly(dimethylsiloxane) (PDMS). This device ("Small Airway-on-a-Chip") contained two parallel microfluidic channels separated by a semiporous extracellular matrix (ECM)-coated membrane that was lined on one side by human primary lung bronchiolar epithelial cells that formed a pseudostratified polarized mucociliated epithelium at ALI and on the opposite side by human pulmonary microvascular endothelial cells that experienced continuous fluid flow (Fig. 1).

The Small Airway Chip was designed so that the top (airway lumen mimic) and the bottom (postcapillary venule mimic) microchannels had similar dimensions to what is observed in vivo. The height of the airway lumen channel, for instance, matched the radius of a human small airway $(1000 \mu \mathrm{m}){ }^{9}{ }^{9}$ The authors applied this reductionist, yet, complex in vitro system to model asthmatic and COPD airway infection and inflammation, to identify diseaseassociated biomarkers, and to test efficacy of experimental drugs. Table 1 summarizes key aspects of this platform and its comparison against airway microphysiological models developed by other groups. ${ }^{30-33}$

In a follow-up study, the same research team elegantly reverses engineered design principles of an average smoker's lung to create a "Breathing-Smoking Human Lung-on-aChip" in vitro (Fig. 1). ${ }^{24,34,35}$ This modular, but microfluidically integrated, system consisted of (1) a microrespirator that simulated diaphragm and rib cage and was capable of cyclically "breathing" microliter volumes of air or smoke in and out of an organ-on-chip microdevice, (2) a Small Airway-on-a-Chip lined by mucociliated human bronchiolar epithelium, (3) a programmable smoking robot that generated fresh whole smoke, and (4) a control software that reproduced human smoking behavior and breathing profilethat is, parameters such as puff duration and volume, interpuff interval, and puffs per cigarette as well as breathing frequency and inhalation-exhalation pattern. This platform enabled the authors to analyze the effects of WCS delivered under physiologically relevant breathing conditions on pathophysiology of human small airway epithelium in a highly controlled manner.

This study (Fig. 2) contributed to significant advances in inhalation toxicology by (1) recapitulating smoke-induced oxidative stress responses of human airway mucosa similar to those observed in respiratory lining of smokers-that is, "functional validation of the system"; (2) identifying novel ciliary dysfunction phenotypes following exposure to WCS - that is, utilizing real-time, high-resolution imaging capacity of organs-on-chips "to detect new micropathologies"; (3) reproducing inhaled smoke-triggered COPD exacerbation and recreating patient-specific responses-that is, applying the platform for "validation and discovery of disease-associated biomarkers" (e.g., selective induction of matrix metalloproteinase-1 $[M M P 1]$, interleukin $8[C X C L 8]$, and transmembrane protease serine 11E [TMPRSS11E] expression with smoking in COPD but not healthy airway epithelia on-chip); and (4) enabling analysis of potential toxic effects of EC vapor-that is, "demonstrating versatility of the platform to study bioimpact of tobacco-related products" in vitro.

A major drawback of human clinical studies, which are the most direct way to assess the effects of exposure to cigarette smoke or any other inhaled particulate, is donor-to-donor variability. The Breathing-Smoking Lung Chip tackles this problem by enabling true matched-comparison of biological responses as the airway cells obtained from the same individual can be cultured in chips and studied in absence or presence of smoke exposure. This greatly enhances chances of distinguishing true signals from noise.

Compared with animal models, the Breathing-Smoking Lung Chip enables study of exposure to "actively" (as opposed to passively) inhaled cigarette smoke on tissues reconstituted using cells obtained from "desired patient (sub)populations" - for example, healthy and COPD, (as opposed to rodent, guinea pig, or rabbits cells) for meaningful clinical and toxicological translation by recreating a well-differentiated airway lining that its cellular distribution is human-relevant - that is, frequency of epithelial subtypes (e.g., basal, club, mucin-producing, and ciliated cells) is closer to the human lung airway. In addition, in comparison with the current state-of-the-art in vitro static smoke exposure systems, the Breathing-Smoking Lung Chip allows recapitulation of smoking and breathing behavior under dynamic breathing airflow conditions.

Two current limitations of the Breathing-Smoking Lung Chip are (1) the absence of subepithelial ECM and stromal cells such as fibroblasts and smooth muscle cells to better mimic organ-level pathophysiology of human airway, and (2) the lack of full assessment of molecular constituentsthat is, dosimetry, of the WCS to more accurately benchmark against known (clinically important) parameters.

Therefore, as this technological platform evolves, it is important to address these aspects, which can be easily achieved by reconfiguring the microchip design, performing gas chromatography/mass spectrometry analyses, and incorporating chemical sensors on-chip. In addition, it would be valuable to embed sensors on-chip for real-time monitoring of partial pressure of oxygen $\left(\mathrm{pO}_{2}\right)$ in the vascular channel and humidity in the airway channel. Integrating the data from $\mathrm{pO}_{2}$ and humidity fluctuations/levels with smoketriggered changes in cellular and tissue function can generate a better picture of molecular mechanisms responsible for pathology development. Finally, the Breathing-Smoking Lung Chip technology needs to be applied to explore toxicopathology of other inhaled compounds and gases.

\section{Conclusion}

In summary, by overcoming several key limitations of static cell cultures and animal models and enabling exposure to whole smoke in a physiologically and clinically relevant context, the "Breathing-Smoking Human Lung-on-a-Chip" offers a great potential for inhalation toxicity studies. While at its infancy, this novel in vitro model provides an entirely new approach to investigate how breathing cigarette smoke influences human lung airway pathophysiology in both normal and diseased individuals. Importantly, it allows discovery of new micropathologies and disease-specific biomarkers as well as comparative toxicoanalysis of different tobacco-related products. 


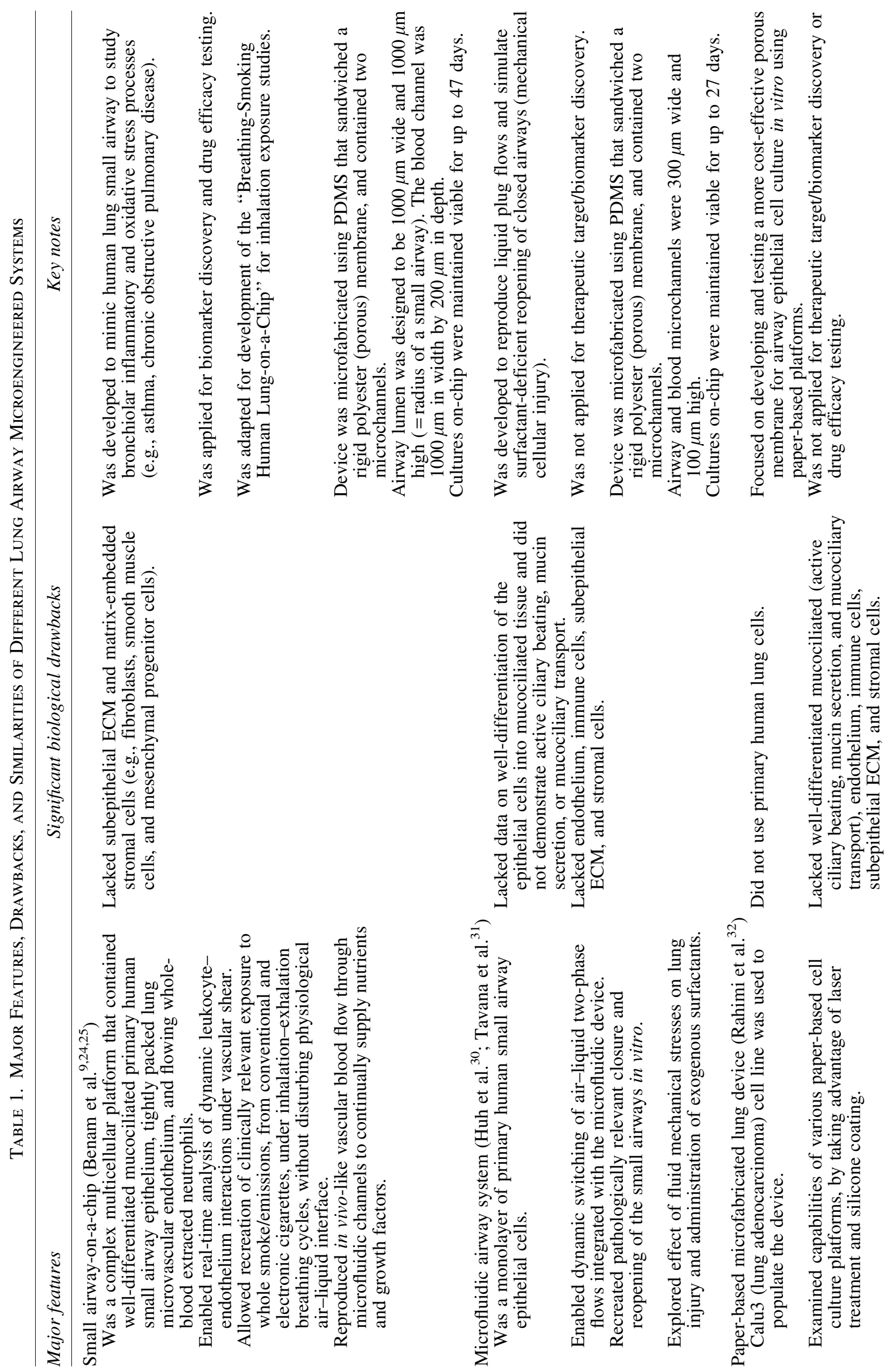




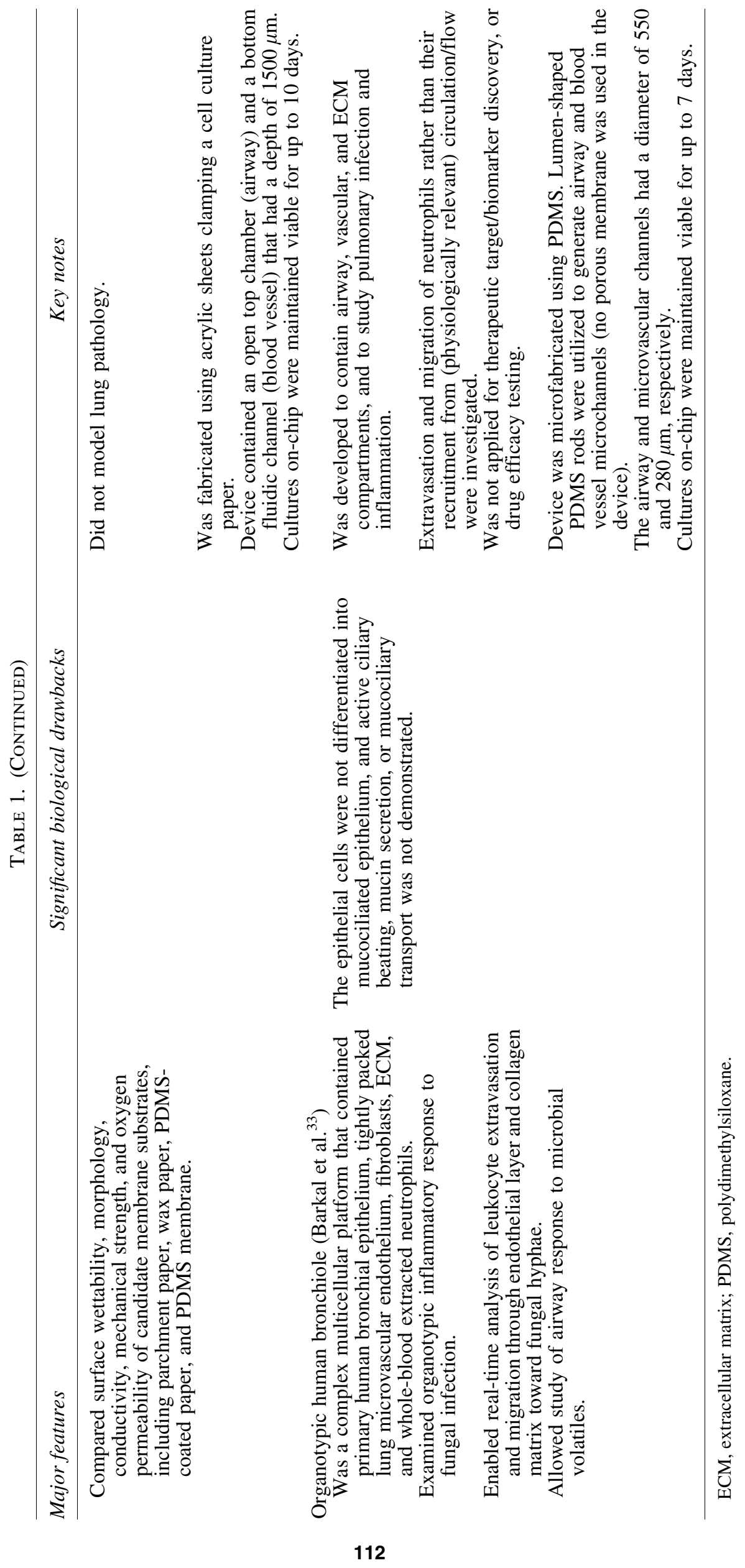



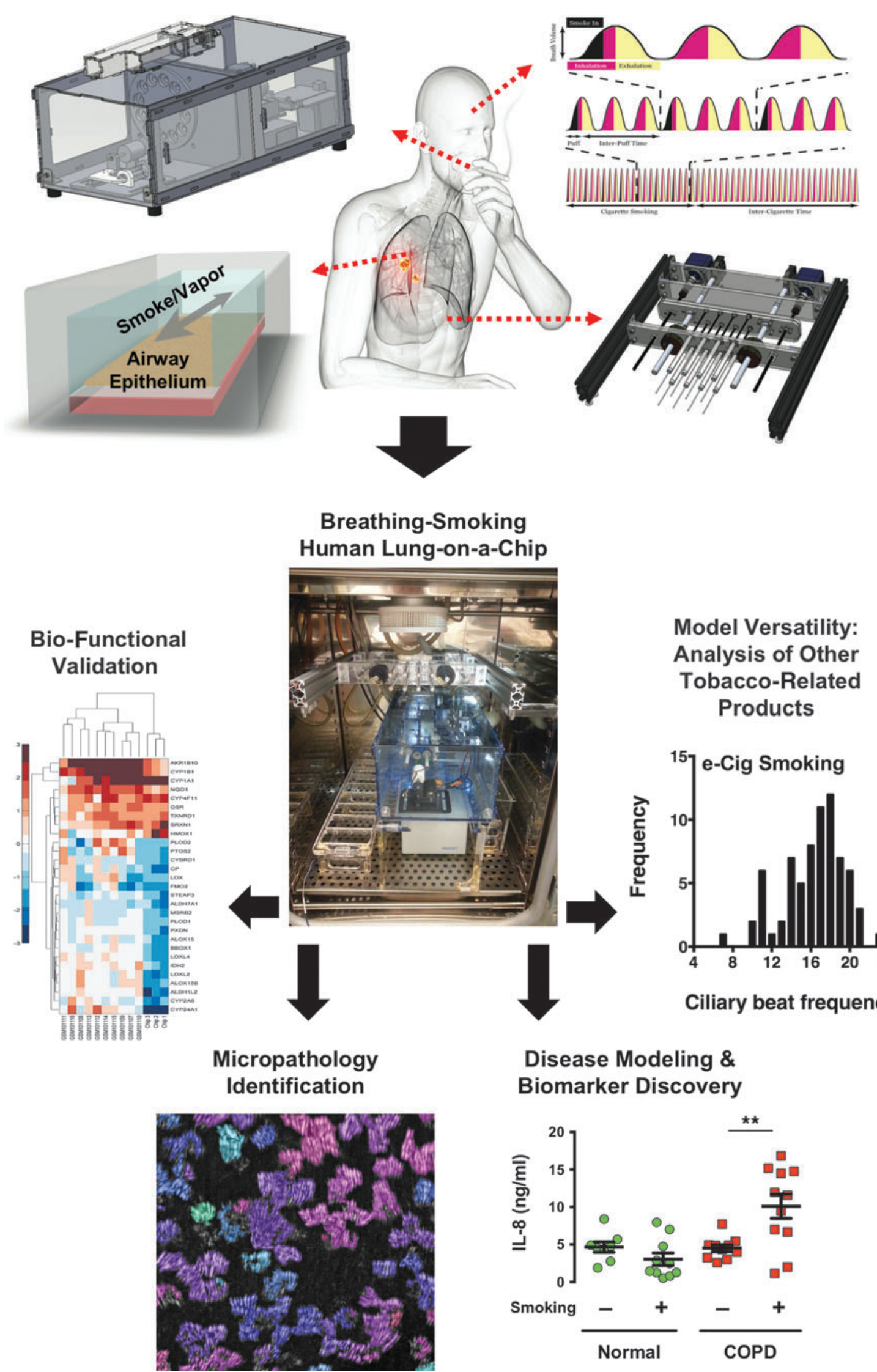

FIG. 2. Microengineered Breathing-Smoking Human Lung-on-a-Chip for toxicoanalysis of inhaled smoke. Top panel: schematic showing the design principle of the components of the Breathing-Smoking Human Lung Chip. Bottom panel: applications of this novel technological platform. Reproduced with modifications from Benam et al. ${ }^{24}$ with permission from Elsevier, Inc. (Cell Press). 


\section{Author Disclosure Statement}

No competing financial interests exist.

\section{References}

1. Phalen RF. Introduction. In: Methods in Inhalation Toxicology. RF Phalens (ed); pp. 1-12. New York: CRC Press, Inc.; 1996.

2. Guirguis-Blake JM, Senger CA, Webber EM, et al. Screening for chronic obstructive pulmonary disease: Evidence report and systematic review for the US preventive services task force. JAMA 2016:315;1378-1393.

3. Mannino DM, Buist AS. Global burden of COPD: Risk factors, prevalence, and future trends. Lancet 2007:370;765-773.

4. Herbst RS, Heymach JV, Lippman SM. Lung cancer. N Engl J Med 2008:359;1367-1380.

5. Neilson L, Mankus C, Thorne D, et al. Development of an in vitro cytotoxicity model for aerosol exposure using 3D reconstructed human airway tissue; application for assessment of e-cigarette aerosol. Toxicol In Vitro 2015:29; 1952-1962.

6. Rowell TR, Tarran R. Will chronic e-cigarette use cause lung disease? Am J Physiol Lung Cell Mol Physiol 2015: 309;L1398-L1409.

7. Wright JL, Cosio M, Churg A. Animal models of chronic obstructive pulmonary disease. Am J Physiol Lung Cell Mol Physiol 2008:295;L1-15.

8. Kolaczkowska E, Kubes P. Neutrophil recruitment and function in health and inflammation. Nat Rev Immunol 2013:13;159-175.

9. Benam KH, Villenave R, Lucchesi C, et al. Small airwayon-a-chip enables analysis of human lung inflammation and drug responses in vitro. Nat Methods 2016:13;151-157.

10. Hecht SS. Carcinogenicity studies of inhaled cigarette smoke in laboratory animals: Old and new. Carcinogenesis 2005:26;1488-1492.

11. Glader P, Moller S, Lilja J, et al. Cigarette smoke extract modulates respiratory defence mechanisms through effects on T-cells and airway epithelial cells. Respir Med 2006: 100;818-827.

12. Mio T, Romberger DJ, Thompson AB, et al. Cigarette smoke induces interleukin-8 release from human bronchial epithelial cells. Am J Respir Crit Care Med 1997:155;1770-1776.

13. Mulligan RM, Atkinson C, Vertegel AA, et al. Cigarette smoke extract stimulates interleukin-8 production in human airway epithelium and is attenuated by superoxide dismutase in vitro. Am J Rhinol Allergy 2009:23; e1-e4.

14. Hellermann GR, Nagy SB, Kong X, et al. Mechanism of cigarette smoke condensate-induced acute inflammatory response in human bronchial epithelial cells. Respir Res 2002:3;22.

15. Scherer G, Conze C, von Meyerinck L, et al. Importance of exposure to gaseous and particulate phase components of tobacco smoke in active and passive smokers. Int Arch Occup Environ Health 1990:62;459-466.

16. Adamson J, Azzopardi D, Errington G, et al. Assessment of an in vitro whole cigarette smoke exposure system: The Borgwaldt RM20S 8-syringe smoking machine. Chem Cent J 2011:5;50.

17. Thorne D, Adamson J. A review of in vitro cigarette smoke exposure systems. Exp Toxicol Pathol 2013:65;1183-1193.

18. Mathis C, Poussin C, Weisensee D, et al. Human bronchial epithelial cells exposed in vitro to cigarette smoke at the airliquid interface resemble bronchial epithelium from human smokers. Am J Physiol Lung Cell Mol Physiol 2013:304; L489-L503.

19. Phillips J, Kluss B, Richter A, et al. Exposure of bronchial epithelial cells to whole cigarette smoke: Assessment of cellular responses. Altern Lab Anim 2005:33;239-248.

20. Lee EM, Malson JL, Waters AJ, et al. Smoking topography: Reliability and validity in dependent smokers. Nicotine Tob Res 2003:5;673-679.

21. Strasser AA, Pickworth WB, Patterson F, et al. Smoking topography predicts abstinence following treatment with nicotine replacement therapy. Cancer Epidemiol Biomarkers Prev 2004:13;1800-1804.

22. Benam KH, Dauth S, Hassell B, et al. Engineered in vitro disease models. Annu Rev Pathol 2015:10;195-262.

23. Bhatia SN, Ingber DE. Microfluidic organs-on-chips. Nat Biotechnol 2014:32;760-772.

24. Benam KH, Novak R, Nawroth $J$, et al. Matchedcomparative modeling of normal and diseased human airway responses using a microengineered breathing lung chip. Cell Syst 2016:3;456-466 e454.

25. Benam KH, Mazur M, Choe Y, et al. Human lung small airway-on-a-chip protocol. Methods Mol Biol 2017:1612; 345-365.

26. Burgel PR, de Blic J, Chanez P, et al. Update on the roles of distal airways in asthma. Eur Respir Rev 2009:18;80-95.

27. Hogg JC, Chu F, Utokaparch S, et al. The nature of smallairway obstruction in chronic obstructive pulmonary disease. N Engl J Med 2004:350;2645-2653.

28. Tiddens HA, Donaldson SH, Rosenfeld M, et al. Cystic fibrosis lung disease starts in the small airways: Can we treat it more effectively? Pediatr Pulmonol 2010:45;107-117.

29. Fulmer JD, Roberts WC, von Gal ER, et al. Small airways in idiopathic pulmonary fibrosis. Comparison of morphologic and physiologic observations. J Clin Invest 1977:60; 595-610.

30. Huh D, Fujioka H, Tung YC, et al. Acoustically detectable cellular-level lung injury induced by fluid mechanical stresses in microfluidic airway systems. Proc Natl Acad Sci U S A 2007:104;18886-18891.

31. Tavana H, Kuo CH, Lee QY, et al. Dynamics of liquid plugs of buffer and surfactant solutions in a micro-engineered pulmonary airway model. Langmuir 2010:26;3744-3752.

32. Rahimi R, Htwe SS, Ochoa M, et al. A paper-based in vitro model for on-chip investigation of the human respiratory system. Lab Chip 2016:16;4319-4325.

33. Barkal LJ, Procknow CL, Alvarez-Garcia YR, et al. Microbial volatile communication in human organotypic lung models. Nat Commun 2017:8;1770.

34. Benam KH, Ingber DE. Commendation for exposing key advantage of organ chip approach. Cell Syst 2016:3;411.

35. Benam KH, Konigshoff M, Eickelberg O. Breaking the in vitro barrier in respiratory medicine: Engineered microphysiological systems for COPD and beyond. Am J Respir Crit Care Med 2017. DOI: 10.1164/rccm.201709-1795PP.

Address correspondence to: Dr. Kambez H. Benam

Division of Pulmonary Sciences and Critical Care Medicine Department of Medicine University of Colorado Anschutz Medical Campus Aurora, CO 80045

E-mail: kambez.benam@ucdenver.edu 